\title{
Interactive comment on "A CMEMS forecasting system for the marine ecosystem of IBI European waters" by Elodie Gutknecht et al.
}

\section{Anonymous Referee \#1}

Received and published: 19 February 2019

\section{General comments:}

The manuscript "A CMEMS forecasting system for the marine ecosystem of IBI" by Elodie Gutknecht et al. aims to describe the skill performances of the CMEMS operational model system "IBI36" for the Iberia-Biscay-Ireland (IBI) area, with specific emphasis on the biogeochemical component. IBI36 is built on a physical-biogeochemical online coupling between NEMOv3.6 physical model and PISCESv2 biogeochemical model, at $1 / 36^{\circ}$ horizontal resolution and with 50 vertical levels. The system is operational since April 2018, and consistently provides 7-days forecasts for ocean physics and biogeochemistry. The validation is applied over the "IBI Extended Domain", and is performed with a 2010-2016 simulation, using a suite of different reference data streams (from satellite and in situ, and 2 BGC-Argo floats), and then on a regional 
basis with comparison with in situ historical data (Northern Seas, North-East Atlantic waters, Bay of Biscay and Mediterranean Sea). IBI36 results generally consistent with the main properties of biogeochemical variables (chlorophyll, nutrients, oxygen and net primary production), in terms of spatial distribution and seasonal cycles. An indicator concerning oxygen deficiency is also proposed and assessed, which may be of great importance for the broad environmental communities, extending to the general public as well.

The manuscript is clear and well written, with a precise subdivision of the different sections (model configuration, reference data and validation, discussion). However, some major weaknesses can be highlighted:

1. The title specifically refers to the "CMEMS forecasting system": even if it may be acknowledged the goal of the work, the validation is here performed at seasonal or annual time scale on the period 2010-2016, computing monthly averages (as defined in P8, L29), and there is no reference to the forecasting skill of the operational system, that should be done on daily (or at least, weekly) averages. So, how the described validation may be suitable for the short-term forecasting system assessment? The reader would be interested in see results on how the IBI36 short-term forecast products compare with reference data (or with the background simulation). Which metrics, of the ones proposed, can then be used operationally to quantitatively evaluate the forecasting skill?

2. The model system products are validated basically following consistency (Figs. 2 to $6,8,9,10 \mathrm{ab}, 11,12,13,15)$, providing BIAS and correlation. However, the uncertainty of products (i.e., forecast, see the previous point) seems not addressed. It may be interesting to quantitatively assess the capability of the model to represent the observed variability: did the author consider to evaluate the model uncertainty, e.g. by means of RMSE? The use of quantitative comparison may be even more useful in the NPP analysis, to estimate the spatial gradient. Further, is there any reason why Fig. 7 does not report the linear regression (as done in Figs. 11 and 14)? Moreover, on a higher

OSD

Interactive comment
Printer-friendly version

Discussion paper

$\mathrm{C} 2$ 
level, given that the validation assessment is discussed following a regional subdivision (which is basically driven by the availability of data), I think it could be useful to provide the readers with a synthesis table with the mean values of the validation metrics (i.e. BIAS, correlation, uncertainty - e.g. as RMSE) per each region.

3. The synthetic overview of the "IBI waters" (Section 2) in terms of the biogeochemical and ecosystem dynamics is interesting since gathers different regions with different properties in a single framework. However, it is not effectively used to define the areas then adopted in the validation (P9, L19). Authors should refer the definition of the 12 small boxes to this overview and then link the different model performances to the different biogeochemical properties of the specific areas. As an example, the availability of reference data may allow validating the consistency of the IBI36 system (or of a specific product) in a specific area/season. And this may be critical to provide indications on the quality of the model system to simulate eutrophication in the different areas (and again, the use of the synthesis table suggested at point 2 would be beneficial for this purpose).

Specific comments:

1. Are there other model applications to the IBI waters (also not operational)? Can the authors discuss how does the IBI36 perform in comparison with other operational systems (e.g. CMEMS Global)? A link with the global model is included only in Section 5 (P15, L15), where some more details (and relevant references) could be added. Also, the comment to the global performance at the eastern boundary (Skagerrak and Kattegat, P12, L16) could be more supported by some reference (again, the CMEMS Global).

2. GODAE Class metrics are widely recognized as references for the skill performance assessment of ocean operational systems and adopted also within the Copernicus community. Is there a specific reason why the authors did not include any mention to them?

Interactive comment

Printer-friendly version

Discussion paper

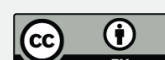


3. Do you have any explanation of why the model anticipates the spring-summer bloom (development and decrease) in the north Atlantic (P9, L14-18)? Is it linked with physical forcing or with the biogeochemical parameterization?

4. Fig. 4 and comment at (P9, L19-24): referring to "high correlation coefficient between the model and the data" I suppose the authors consider correlation values ( $r$ ) larger than 0.7. However, box 12 (Gulf of Cadiz) has a correlation $r=0.55$, which is actually lower than the one for the English Channel (box 6, $r=0.59$ ). Please comment. Further in Fig. 4: how the associated error to ESA OC-CCI product is estimated?

5. Which specific products have been used from EMODnet? Please clarify the kind of "regional aggregated products" used (P7, L31-33). Further, you refer to EMODnet database as "in situ" (P10, L23; P13, L10, and P14, L25), but this looks in contradiction with the "aggregation" previously referred. Please clarify and reformulate.

6. Fig. 10 is potentially very interesting, especially for communities more linked to environmental management. I suggest to enrich the discussion and provide some more information on this indicator: how is computed the minimum in Fig.10a and b (is this defined considering at least 1 daily value lower than the threshold as in P11, L17? is the minimum found along the vertical profile, or at the sea bottom? That should be defined at the beginning of the discussion at P11)? Do the two maps show the absolute minimum over the whole investigated period for each cell shown? What is shown in the time series of Fig.10c? Please clarify. Further, the deficiency threshold (at $187 \mathrm{umol} / \mathrm{l}$ ) could be added to Fig.10c (e.g. with a dashed line): this may help to appreciate the model skill in representing the oxygen deficiency, and the fact that in 2011 and 2015 the model predicts higher levels than observed. To measure the skill of the model to overcome a specific threshold in comparison with observed data, authors may also consider using the statistics based on the relative operating characteristic (ROC), as for example shown in Sheng and Kim (J. Mar. Sys. 76, 212-243, 2009).

7. Figs. 14 and 15 show the comparison with BGC-Argo floats data in Atlantic and West

Interactive comment
Printer-friendly version

Discussion paper 
Mediterranean areas covered by the IBI system. May the authors add any comment on the systematic behavior of the model in comparison with the float also for nitrate OSD and oxygen (as done for Chl-a at depth, see P14, L2, and then in the discussion, P15, L13)? Do the authors suspect that this behavior is related to possible errors of the model (due to representativeness or biogeochemical parameterization), or may be also related to uncertainty associated to float sensors? Further, does the white line in Fig. 15 represent the MLD? If so, it should be added to the caption, and briefly explained how it was computed.

8. In the discussion (P15, L1-6) why do the authors write that the higher biases on the continental shelf are "no surprising"? Is this related to larger uncertainty given by the river input? Or is there any limits due to the physical drivers? If it seems obvious, please clarify. Secondly, is there any literature basis for the NPP (e.g. with in situ measurements) that can be used to establish which of the 3 referred products we should trust more?

9. At the end of the discussion, there is a reference to OMI (P16, L8-11): this might not be interesting for the general reader. However, since there is a specific indication of the "IBI-MFC biogeochemical forecast service", this should be properly validated before providing OMI (see major point n.1).

\section{Technical/other corrections:}

In the webpage of OSD (https://www.ocean-sci-discuss.net/os-2018-161/\#discussion), the correct author's surname should be "Sotillo" and not "Sottilo".

P2, L8 and P4, L31. The "IBI-MFC Team" has not been defined.

P3, L14-15. "The annual primary production is then limited its seasonal variations are limited": not clear, please correct or reformulate.

P3, L29. References about N:P ratio around 20 in the Western Mediterranean are also found in the recent work of Lazzari et al. (Deep-Sea Res. I 108, 39-52, 2016; see their

Interactive comment

Printer-friendly version

Discussion paper 
Fig. 7), that may be added.

P5, L25-27. "Although PISCES was originally designed for global ocean applications, OSD the distinction of two phytoplankton size classes and the description of multiple nutrient co-limitations allow the model to represent ocean productivity and biogeochemical cycles in the major ocean biogeographic provinces (Longhurst, 1998)." The reference seems not appropriate: PISCES is not used in Longhurst (1998), which only refers to the bio-provinces. Please reformulate.

P6, L1-2. "To respect the conservation of the tracers, the coupling between biogeochemical and physical components is done every other time." Not clear, please clarify: what do the authors consider as "conservation of the tracers"? Is it mass conservation? This question also relates to the reference to Aumont et al. (2015), at L19-L34: it would be clearer for the reader to add more details about the river inputs (which are very important in coastal regions). Please describe the specific inputs (also reporting from Aumont, Section 4.9.2, and the one from EEA), list the names of the rivers (or their total amount), specify what you consider as "natural" and what as "anthropic", and describe their impact in the investigated region. Finally, please clarify the use of "reminder" the last sentence "For the other variables, a reminder of the initial conditions is given.": does this mean the initial condition values are maintained constant during the simulation?

P6, L8. "is" should be "are".

P7, L20. As done with VGPM and CbPM, please add a reference also to the "Eppley version".

P8, L6-18. Could you provide which fields from the BGC-Argo repository have been used and whether additional calibrations were performed after?

Printer-friendly version

P8, L18. "Johnson et al." (dot missing).

Discussion paper

P11, L13. "Breitburg et al." (dot missing).

Interactive

comment 
P11, L19. It should be "surface area in winter".

P11, L20. The authors refer to "the west coast of France" but in Fig.10 a and b it looks OSD like only the north-western French coast is covered by data, and with value larger than 200 umol/l. Please check.

$\mathrm{P} 12$, L4. "The seasonal cycle is in phase for Chl-a but out of phase for ammonium" it

Interactive is also possible to refer to Fig.7e, where correlation for $\mathrm{NH} 4$ is $\mathrm{r}=0.2$.

comment

P14, L25. Instead of "is described here", it should be clearer to indicate the corresponding Section or Figure (Figs. 2, 4 for Chl-a and 5, 6 for NPP). Or are the authors here referring to the general approach of the manuscript? Please clarify. Further, it should be "are described".

P17, L3. The fact that a co-author is also acknowledged sounds somehow strange. Please check.

P29, L3. Correct "Eppley".

P33, Fig. 10. Add the units for O2 minimum (umol/l).

Interactive comment on Ocean Sci. Discuss., https://doi.org/10.5194/os-2018-161, 2019. 